\title{
Espirometria de incentivo a volume versus a fluxo sobre parâmetros respiratórios em idosos
}

\author{
Incentive spirometry volume-oriented versus flow-oriented \\ on respiratory parameters in elderly people
}

Espirometría de incentivo a volumen versus a flujo sobre parámetros respiratorios en añosos

Fernanda dos Santos Pascotini', Mônica de Castro Ramos², Antônio Marcos Vargas da Silva', Maria Elaine Trevisan ${ }^{1}$

RESUMO I No processo de envelhecimento, o indivíduo passa por mudanças fisiológicas, destacando-se o declínio da função pulmonar. A espirometria de incentivo, utilizada em diversas populações, ainda não deixa clara a diferença entre os métodos a volume e a fluxo sobre variáveis respiratórias em sujeitos idosos. O objetivo do estudo foi comparar os efeitos da espirometria de incentivo - volume e fluxo, sobre a função pulmonar, força muscular respiratória e mobilidade tóraco-abdominal de idosos saudáveis. Trata-se de um ensaio clínico com 48 idosos, entre 60 e 84 anos de idade, randomizados para espirometria de incentivo a volume ( $n=23$ ) ou a fluxo ( $n=25)$. Foram avaliadas as pressões inspiratória (PImáx) e expiratória (PEmáx) máximas, volumes e capacidades pulmonares e cirtometria tóraco-abdominal pré e pós-treinamento domiciliar. Os dados foram analisados pela análise de variância de duas vias com medidas repetidas. Houve aumento da PImáx, PEmáx, capacidade vital forçada (CVF), volume expiratório forçado no primeiro segundo, volume minuto, volume corrente e cirtometria a nível xifoide e umbilical em ambos os grupos ( $p<0,001)$. Na comparação entre os grupos, o aumento na CVF foi maior no grupo fluxo $(p=0,03)$ e maior cirtometria axilar no grupo volume $(p=0,02)$. Ambos os incentivadores foram eficazes na melhora da função pulmonar, força muscular respiratória e mobilidade tóraco-abdominal em idosos saudáveis, mostrando-se bons aliados da fisioterapia respiratória.

Descritores I Exercícios Respiratórios; Músculos Respiratórios; Medidas de Volume Pulmonar;

Envelhecimento; Fisioterapia.
ABSTRACT I In the aging process, the individual goes through physiological changes, especially the decline in lung function. The incentive spirometry, used in many populations, not yet makes clear the difference between the methods and the volume flow on respiratory variables in older subjects. This study aimed to compare the effects of incentive spirometry - volume and flow, on pulmonary function, respiratory muscle strength and thoracoabdominal mobility in healthy elderly. It was a clinical trial with 48 elderly between 60 and 84 years old, randomized to the incentive spirometry by volume $(n=23)$ or by flow ( $n=25)$. We evaluated the maximum inspiratory pressure (MIP) and the maximum expiratory pressure (MEP), volumes and pulmonary capacities and thoracoabdominal cirtometry before and after home training. The data were analyzed by two-way repeated measures analysis of variance. There was an increase in MIP, MEP, forced vital capacity (FVC), forced expiratory volume in one second, minute volume, tidal volume and xiphoid and umbilical cirtometry level in both groups ( $p<0.001)$. When comparing both groups, the increase in FVC was higher in flow group ( $p=0.03$ ) and there was a greater increase in axillary cirtometry in volume group ( $p=0.02$ ). Both incentives were effective in improving lung function, respiratory muscle strength and thoracoabdominal mobility in healthy elderly, being good allies of respiratory therapy.

Keywords I Breathing Exercises; Respiratory Muscles; Lung Volume Measurements; Aging; Physical

Therapy Specialty.

Estudo desenvolvido no Departamento de Fisioterapia e Reabilitação da Universidade Federal de Santa Maria (UFSM) - Santa Maria (RS), Brasil.

UUFSM - Santa Maria (RS), Brasil.

¿Universidade Luterana do Brasil (ULBRA) - Santa Maria (RS), Brasil. 
RESUMEN I En el proceso de envejecimiento, el individuo pasa por cambios fisiológicos, destacándose la declinación de la función pulmonar. La espirometría de incentivo, utilizada en diversas poblaciones, aun no deja clara la diferencia entre los métodos a volumen y a flujo sobre variables respiratorias en sujetos añosos. El objetivo fue comparar los efectos de la espirometría de incentivo - volumen y flujo, sobre la función pulmonar, fuerza muscular respiratoria y movilidad tóraco-abdominal de añosos sanos. Este fue un ensayo clínico con 48 añosos, entre 60 y 84 años de edad, randomizados para espirometría de incentivo a volumen ( $n=23$ ) o a flujo ( $n=25)$. Fueron evaluadas las presiones inspiratoria (PImáx) y expiratoria (PEmáx) máximas, volúmenes y capacidades pulmonares y cirtometría tóraco-abdominal pre y post entrenamiento domiciliario. Los datos fueron analizados por el análisis de varianza de dos vías con medidas repetidas. Hubo aumento de la PImáx, PEmáx, capacidad vital forzada (CVF), volumen expiratorio forzado en el primer segundo, volumen minuto, volumen corriente y cirtometría a nivel xifoides y umbilical en ambos grupos ( $p<0,001$ ). En la comparación entre los grupos, el aumento en la CVF fue mayor en el grupo flujo $(p=0,03)$ y mayor cirtometría axilar en el grupo volumen ( $p=0,02$ ). Ambos incentivadores fueron eficaces en la mejora de la función pulmonar, fuerza muscular respiratoria y movilidad tóraco-abdominal en añosos sanos, mostrándose buenos aliados de la fisioterapia respiratoria.

Palabras clave I Ejercicios Respiratorios; Músculos

Respiratorios; Medidas del Volumen Pulmonar;

Envejecimiento; Fisioterapia.

\section{INTRODUÇÃO}

Em decorrência da aceleração no ritmo de crescimento da população idosa nos países em desenvolvimento ${ }^{1}$, prevêse que em 2025 cerca de 14\% da população brasileira seja composta por idosos ${ }^{2}$. O envelhecimento saudável é natural e progressivo, com mudanças funcionais gradativas que permitem adaptações e manutenção da qualidade de vida ${ }^{3}$.

A função pulmonar diminui lentamente após atingir a vida adulta ${ }^{4}$ e, em decorrência do envelhecimento, ocorre redução da mobilidade da caixa torácica, elasticidade pulmonar, força muscular respiratória e capacidade vital, ocasionando redução da eficiência da tosse e da mobilidade ciliar no epitélio respiratório 5 . Há redução do volume pulmonar, aumento do volume residual, fechamento precoce de pequenas vias aéreas, redução da complacência torácica e aumento da complacência pulmonar, entre outras alterações ${ }^{6}$.

Diante destes ajustes fisiológicos, a fisioterapia tem o papel de retardar ou eventualmente recuperar a inevitável perda de função pulmonar do idoso. Diversas estratégias terapêuticas podem oferecer benefícios, tanto pelo uso de padrões ventilatórios voluntários quanto por equipamentos específicos. A espirometria de incentivo consiste na utilização de equipamentos projetados para estimular inspirações profundas, lentas e sustentadas, com estímulo visual, podendo ser orientados a volume ou fluxo ${ }^{7,8}$.

Estudos relacionados ao exercício respiratório geralmente abordam portadores de patologias cardiorrespiratórias crônicas e poucos enfocam a população idosa sem patologias ${ }^{9}$. Diante do crescimento da população idosa, deterioração do sistema respiratório com o avanço da idade e necessidade de avaliar novas estratégias terapêuticas, este estudo objetivou comparar os efeitos da espirometria de incentivo a volume e a fluxo sobre a função pulmonar e força muscular respiratória de idosos saudáveis.

\section{METODOLOGIA}

Pesquisa aprovada pelo Comitê de Ética em Pesquisa da Universidade Federal de Santa Maria, CAAE 0356.0.243.000-11. Todos os participantes assinaram o Termo de Consentimento Livre e Esclarecido.

Incluídos idosos de ambos os sexos, com idade entre 60 e 90 anos, sem diagnóstico prévio de patologias respiratórias. Excluídos idosos com doenças neurológicas e/ou psiquiátricas, sintomas de resfriado e/ou afecções respiratórias no momento da avaliação, etilista, tabagista atual e ex-tabagista há menos de dez anos, cirurgia abdominal e torácica há menos de cinco anos.

Amostra constituída por 48 indivíduos recrutados por divulgação na mídia, randomizados por sorteio em grupo Voldyne $^{\varpi}$ (GVold) e grupo Respiron ${ }^{\circledast}$ (GResp). A força muscular respiratória, mobilidade tóraco-abdominal e função pulmonar foram verificadas pré e pós-treinamento.

A força muscular respiratória foi verificada pelo manovacuômetro digital Microhard MVD500 (Globalmed Porto Alegre/RS), com os participantes sentados, com clipe nasal e bocal firme entre os lábios. Foram realizadas duas manobras de aprendizado e combinado o gesto manual que indicaria quando os pulmões estivessem inflados/desinflados.

Para medir a pressão inspiratória máxima (PImáx) solicitou-se expiração em nível de Volume Residual (VR), seguida de uma inspiração rápida e forte em nível da Capacidade Pulmonar Total (CPT) sustentada por um segundo, com estímulo verbal do examinador. Para a pressão expiratória máxima (PEmáx) solicitou-se inspiração máxima em nível da CPT seguida de uma expiração máxima até o nível do VR, mantendo-a por um segundo, com estímulo verbal do examinador ${ }^{10}$. Foram efetuadas cinco manobras máximas, com intervalo de um minuto de descanso 
e, posteriormente, selecionadas três manobras aceitáveis e reprodutíveis (diferença de $10 \%$ ou menos entre os esforços), sendo registrado o valor mais alto ${ }^{11}$ e comparado ao valor predito pela equação de Neder $\mathrm{et} a \mathrm{l} .{ }^{12}$, de acordo com idade e sexo.

Para medir a expansibilidade tóraco-abdominal pela cirtometria foram utilizadas três fitas métricas, adaptadas com uma alça em cadarço de algodão para servir de guia no deslizamento das fitas durante os movimentos respiratórios. Em decúbito dorsal, as fitas foram posicionadas em três pontos anatômicos de referência prega axilar, apêndice xifoide e linha umbilical e as medidas realizadas em repouso, após inspiração máxima (CPT) e após expiração máxima (VR), sob o comando do pesquisador. Para cada ponto foram realizadas três medidas, nos três diferentes momentos com intervalos de um minuto entre elas ${ }^{13}$.

O Volume Corrente (VC), Frequência Respiratória (FR), Volume Minuto (VM), Capacidade Vital Forçada (CVF) e Volume Expiratório Forçado no Primeiro Segundo $\left(\mathrm{VEF}_{1}\right)$ foram obtidos pelo espirômetro Respiradyne II (Model 5-7930P Sher Wood Medical Co). Para o VC, FR e VM, o sujeito deveria respirar normalmente no bocal do aparelho por um minuto. A CVF e o $\mathrm{VEF}_{1}$ foram obtidos a partir da inspiração máxima, seguida da expiração rápida e sustentada no bocal do aparelho, por pelo menos 6 segundos. Houve estímulo para que o esforço fosse "explosivo" no início da manobra, repetida até se obter três manobras aceitáveis e reprodutíveis ${ }^{14}$. Todas as medidas foram obtidas na posição sentada, com uso de clipe nasal.

Treinamento realizado no domicílio com supervisão direta do pesquisador, uma sessão diária, por 12 dias consecutivos. O protocolo de treinamento foi o mesmo para ambos os grupos, exceto o tipo de incentivador. Foram orientados a realizar uma inspiração lenta a partir da Capacidade Residual Funcional (CRF) até atingir o nível previamente demarcado no cilindro do Voldyne (GVold) ou pelo anel regulador (GResp) sustentada no final por um segundo ${ }^{15}$. O protocolo foi elaborado pelos pesquisadores e contou com a seguinte sequência: 3 séries de 8 repetições nos três primeiros dias, 3 séries de 10 repetições do $4^{\circ}$ ao $6^{\circ}$ dia, 3 séries de 12 repetições do $7^{\circ}$ ao $9^{\circ}$ dia e 3 séries de 14 repetições do $10^{\circ}$ ao $12^{\circ}$ dia. Indivíduos na posição sentada, incentivo verbal para melhor execução da técnica, padrão muscular diafragmático previamente treinado, intervalo de cinco minutos entre as séries para descanso.

$\mathrm{Na}$ análise estatística a comparação entre os grupos ocorreu pelo teste qui-quadrado (variáveis categóricas) ou pelo teste $t$ de Student não pareado (variáveis contínuas).
A comparação intra e entre grupos ocorreu pela Análise de Variância (duas vias) com medidas repetidas e analisou três efeitos (tempo, grupo, interação), seguida do post hoc de Bonferroni, em nível de significância de 5\%. Foi utilizado o programa Statistical Package for the Social Sciences (SPSS) versão 13.0. Cálculo amostral baseado no estudo de Burneiko et al. ${ }^{16}$, para obtenção de um nível de significância de 5\% e poder (beta) de 80\%, sendo estimado 23 sujeitos em cada grupo.

\section{RESULTADOS}

Participaram do estudo 48 idosos, com idade entre 60 e 84 anos, randomizados em GResp ( $\mathrm{n}=25)$ e GVold ( $\mathrm{n}=23$ ). Os grupos foram homogêneos $(p>0,05)$ em relação às variáveis antropométricas, espirométricas, força muscular respiratória e expansibilidade tóraco-abdominal (Tabela 1).

A comparação intra e entre grupos, nos períodos pré e pós-intervenção, é apresentada na Tabela $2 . \mathrm{Na}$ comparação intragrupo, analisado pelo efeito tempo, evidenciou-se aumento em ambos os grupos na PImáx (Figura 1A), PEmáx (Figura 1B), CVF (Figura 1C), $\mathrm{VEF}_{1}$ (Figura 1D), VM (Figura 1E), VC (Figura 1F), cirtometria xifoide e umbilical. A cirtometria axilar e a FR não diferiram entre pré e pós-intervenção. $\mathrm{Na}$

Tabela 1. Características basais dos grupos

\begin{tabular}{|c|c|c|c|}
\hline \multirow{2}{*}{ Variáveis } & GResp & GVold & \multirow{2}{*}{ Valor $\mathrm{p}$} \\
\hline & $(n=25)$ & $(n=23)$ & \\
\hline Idade (anos) & $69,9 \pm 6,6$ & $69,2 \pm 7,8$ & 0,72 \\
\hline Sexo (F/M) & $20 / 5$ & $15 / 8$ & 0,21 \\
\hline $\mathrm{IMC}\left(\mathrm{kg} / \mathrm{m}^{2}\right)$ & $25,5 \pm 3,2$ & $26,1 \pm 5$ & 0,65 \\
\hline PImáx $\left(\mathrm{cm} / \mathrm{H}_{2} \mathrm{O}\right)$ & $51,4 \pm 12,6$ & $56,7 \pm 19,7$ & 0,27 \\
\hline PImáx (\% predito) & $64,1 \pm 15,6$ & $67,3 \pm 22,5$ & 0,57 \\
\hline PEmáx $\left(\mathrm{cm} / \mathrm{H}_{2} \mathrm{O}\right)$ & $60,4 \pm 14,3$ & $65,4 \pm 24,8$ & 0,40 \\
\hline PEmáx (\% predito) & $77,1 \pm 17,9$ & $76,7 \pm 25,9$ & 0,96 \\
\hline CVF (L) & $2,2 \pm 0,5$ & $2,5 \pm 0,7$ & 0,07 \\
\hline $\operatorname{VEF}_{1}(\mathrm{~L})$ & $1,8 \pm 0,4$ & $1,9 \pm 0,6$ & 0,62 \\
\hline VM (L/min) & $6,9 \pm 2$ & $7 \pm 1,5$ & 0,85 \\
\hline VC (ml) & $527,3 \pm 103$ & $506 \pm 106$ & 0,48 \\
\hline FR (rpm) & $13,3 \pm 2,7$ & $13,9 \pm 2,3$ & 0,38 \\
\hline $\mathrm{CA}(\mathrm{cm})$ & $4,3 \pm 1,8$ & $3,9 \pm 1,6$ & 0,40 \\
\hline $\mathrm{CX}(\mathrm{cm})$ & $3,5 \pm 1,3$ & $3 \pm 1,3$ & 0,18 \\
\hline $\mathrm{CU}(\mathrm{cm})$ & $3,7 \pm 0,9$ & $3,3 \pm 1,3$ & 0,21 \\
\hline
\end{tabular}

GVold: grupo Voldyne; GResp: grupo Respiron`; M: masculino; F: feminino; IMC: índice de massa corporal; PImáx: pressão inspiratória máxima; PEmáx: pressão expiratória máxima; CVF: capacidade vital forçada; $V E F$ : volume expiratório forçado no primeiro segundo; $V M$ : volume-minuto; VC: volume corrente; FR: frequência respiratória; CA: cirtometria axilar; CX: cirtometria xifoidiana; CU: cirtometria umbilical; *diferença significante $(p<0,05)$ 
Tabela 2. Força muscular respiratória, função pulmonar e cirtometria tóraco-abdominal pré e pós-intervenção

\begin{tabular}{|c|c|c|c|c|c|c|}
\hline \multirow{2}{*}{ Variáveis } & \multirow{2}{*}{ Período } & \multirow{2}{*}{ GResp } & \multirow{2}{*}{ GVold } & \multicolumn{3}{|c|}{ ANOVA (valor $p$ ) } \\
\hline & & & & Tempo & Grupo & Interação \\
\hline \multirow{2}{*}{ PImáx (cm/ $\left.\mathrm{H}_{2} \mathrm{O}\right)$} & Pré & $51,4 \pm 12,6$ & $56,7 \pm 19,7$ & \multirow{2}{*}{$<0,001^{*}$} & \multirow{2}{*}{0,18} & \multirow{2}{*}{0,82} \\
\hline & Pós & $61,3 \pm 15$ & $65,9 \pm 19$ & & & \\
\hline \multirow{2}{*}{ PImáx (\% do predito) } & Pré & $64,1 \pm 15,6$ & $67,3 \pm 22,5$ & \multirow{2}{*}{$<0,001^{*}$} & \multirow{2}{*}{0,77} & \multirow{2}{*}{0,63} \\
\hline & Pós & $77,6 \pm 17,3$ & $78,4 \pm 21,9$ & & & \\
\hline \multirow{2}{*}{ PEmáx $\left(\mathrm{cm} / \mathrm{H}_{2} \mathrm{O}\right)$} & Pré & $60,4 \pm 14,3$ & $65,4 \pm 24,8$ & \multirow{2}{*}{$<0,001^{*}$} & \multirow{2}{*}{0,31} & \multirow{2}{*}{0,89} \\
\hline & Pós & $68,1 \pm 14$ & $73 \pm 24,6$ & & & \\
\hline \multirow{2}{*}{ PEmáx (\% do predito) } & Pré & $77,1 \pm 17,9$ & $76,7 \pm 25,9$ & \multirow{2}{*}{$<0,001^{*}$} & \multirow{2}{*}{0,63} & \multirow{2}{*}{0,90} \\
\hline & Pós & $91,4 \pm 18,8$ & $88,5 \pm 28,9$ & & & \\
\hline \multirow{2}{*}{ CVF (L) } & Pré & $2,2 \pm 0,5$ & $2,5 \pm 0,7$ & \multirow{2}{*}{$<0,001^{*}$} & \multirow{2}{*}{0,26} & \multirow{2}{*}{$0,03^{*}$} \\
\hline & Pós & $2,6 \pm 0,6$ & $2,7 \pm 0,8$ & & & \\
\hline \multirow{2}{*}{$\operatorname{VEF}_{1}(\mathrm{~L})$} & Pré & $1,8 \pm 0,4$ & $1,9 \pm 0,6$ & \multirow{2}{*}{$<0,001^{*}$} & \multirow{2}{*}{0,83} & \multirow{2}{*}{0,49} \\
\hline & Pós & $2,2 \pm 0,6$ & $2,2 \pm 0,7$ & & & \\
\hline \multirow{2}{*}{ VM (L/min) } & Pré & $6,9 \pm 2$ & $7,04 \pm 1,5$ & \multirow{2}{*}{$<0,001^{*}$} & \multirow{2}{*}{0,84} & \multirow{2}{*}{0,65} \\
\hline & Pós & $8,2 \pm 2,2$ & $8,4 \pm 2$ & & & \\
\hline$V C(m L)$ & Pré & $527,3 \pm 103$ & $506 \pm 106$ & $<0001 *$ & 040 & 074 \\
\hline Ve (IIL) & Pós & $692 \pm 127$ & $652 \pm 140$ & $<, \cup \cup 1$ & 0,40 & 0,14 \\
\hline FR (rom) & Pré & $13,3 \pm 2,7$ & $13,9 \pm 2,3$ & 015 & ০८० & 015 \\
\hline $5 \pi(1011)$ & Pós & $14,4 \pm 1,9$ & $13,7 \pm 2$ & 0,13 & 0,00 & 0 \\
\hline $\mathrm{CA}(\mathrm{cm})$ & Pré & $4,3 \pm 1,8$ & $3,9 \pm 1,6$ & 072 & 0.66 & $0.02 *$ \\
\hline & Pós & $3,9 \pm 1,1$ & $4,6 \pm 1,6$ & & 0,06 & $0, U<$ \\
\hline & Pré & $3,5 \pm 1,3$ & $3 \pm 1,3$ & & & \\
\hline (x (cm) & Pós & $4,6 \pm 1,4$ & $4,6 \pm 1,9$ & $<0,001^{x}$ & 0,49 & 0,30 \\
\hline $\mathrm{CU}(\mathrm{cm})$ & Pré & $3,7 \pm 0,9$ & $3,3 \pm 1,3$ & & 043 & 079 \\
\hline cu (cाII) & Pós & $4,8 \pm 1,9$ & $4,5 \pm 1,7$ & $<, 001$ & 0,43 & 0,19 \\
\hline
\end{tabular}

GResp: grupo Respiron`; GVold: grupo Voldyne; PImáx: pressão inspiratória máxima; PEmáx: pressão expiratória máxima; CVF: capacidade vital forçada; VEF 1 volume expiratório forçado no primeiro segundo; VM: volume-minuto; VC: volume corrente; FR: frequência respiratória; CA: cirtometria axilar; CX: cirtometria xifoidiana; CU: cirtometria umbilical; *diferença significante (p<0,05)
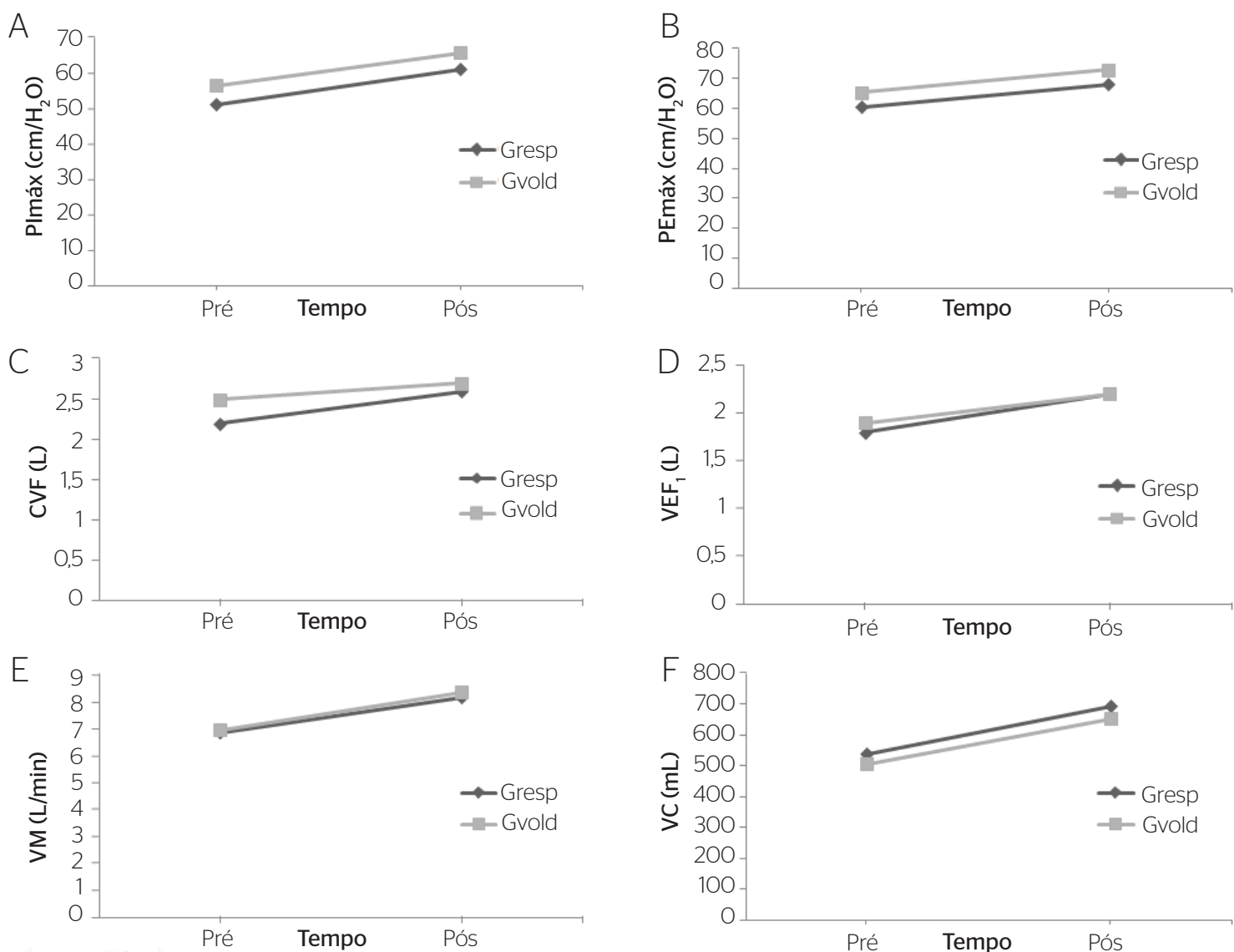

PImáx: pressão inspiratória máxima; PEmáx: pressão expiratória máxima; CVF: capacidade vital forçada; VEF, volume expiratório forçado no primeiro segundo; VM: volume-minuto; VC: volume corrente; GResp: grupo Respiron"

Figura 1. Valores apresentados em médias. Variações da pressão inspiratória máxima (A); variações da pressão expiratória máxima (B); variações da capacidade vital forçada (C); variações do volume expiratório forçado no primeiro segundo (D); variações do volume-minuto (E); variações do volume corrente (F) 
comparação entre grupos, o GResp apresentou maior incremento na CVF (efeito interação) e o GVold apresentou maior elevação na cirtometria axilar (efeito interação). Os grupos não diferiram entre si para as demais variáveis na análise dos efeitos grupo e interação.

\section{DISCUSSÃO}

Após treinamento a CVF aumentou em ambos os grupos, o que corrobora com um estudo anterior ${ }^{17}$ que comparou os volumes pulmonares após o uso do Respiron $^{\circledast}$ e do Voldyne ${ }^{\circledast}$ no pós-operatório de cirurgia abdominal alta. Não foram encontrados na literatura, até o momento, relatos envolvendo melhora da CVF em idosos saudáveis após tratamento com espirometria de incentivo, sendo este um dos principais achados de nosso estudo, o qual se mostra propositivo ao apresentar ferramentas terapêuticas adicionais para esta população. Além disso, o acréscimo da CVF foi maior com a utilização do incentivador a fluxo, o que sugere que o fluxo rápido e turbulento gerado pode proporcionar maiores benefícios em variáveis que envolvem manobras de respiração forçada.

Neste estudo, os idosos apresentaram pressões respiratórias máximas abaixo dos valores preditos para a idade $^{12}$, o que corrobora com estudos ${ }^{18-22}$ que mostram que a força muscular respiratória apresenta-se diminuída em idosos. Após treinamento pôde-se observar aumento significativo da PImáx e da PEmáx em ambos os grupos, sugerindo que estas terapêuticas foram eficazes, como observado anteriormente por Kotz ${ }^{23}$, porém envolvendo jovens saudáveis.

A literatura é escassa quando se trata dos efeitos da espirometria de incentivo na população saudável. Weindler e Kiefer ${ }^{24}$ observaram acréscimo significativo na PImáx após terapia com espirômetros orientados a fluxo e a volume em pacientes submetidos à cirurgia abdominal e/ou cardíaca. Estudo recente ${ }^{25}$ comparou os efeitos da espirometria de incentivo a fluxo e a volume sobre os volumes pulmonares, mobilidade tóraco-abdominal e atividade muscular inspiratória em idosos e em adultos saudáveis, concluindo que ambos os espirômetros têm efeitos semelhantes nos volumes pulmonares e mobilidade tóraco-abdominal, porém o espirômetro a fluxo exige maior atividade muscular respiratória.

Os achados desta pesquisa reforçam a hipótese de que a espirometria de incentivo, mesmo não tendo como alvo terapêutico específico a melhora da força muscular respiratória, pode exercer efeitos positivos na performance muscular respiratória e poderá ser oferecida como estratégia no tratamento de sujeitos com fraqueza muscular.

O grau de mobilidade torácica abaixo dos parâmetros da normalidade foi também evidenciado por Guimarães et al. ${ }^{26}$. Após a intervenção houve aumento significante no diâmetro em nível do xifoide e linha umbilical em ambos os grupos. A normalidade da mobilidade torácica em um adulto jovem saudável é em torno de $7 \mathrm{~cm}^{26}$, sendo este valor reduzido em idosos, devido às modificações da estrutura torácica ${ }^{26}$. A expansibilidade em nível axilar foi maior no GVold que no GResp, o que pode sugerir uma maior distribuição do fluxo aéreo em lobos superiores com o incentivador a volume em comparação ao de fluxo. No entanto, não se pode afirmar que este fenômeno está efetivamente relacionado à distribuição de fluxo aéreo, pois este estudo não envolveu técnicas para mensuração dessa variável. $\mathrm{O}$ aumento da expansibilidade tóraco-abdominal também foi relatado em estudo prévio, porém em pacientes com doença pulmonar crônica, o qual observou que o incentivador a volume obteve melhores resultados quando comparado ao incentivo a fluxo ${ }^{27}$.

Evidenciou-se o aumento no VC em ambos os grupos, mostrando a efetividade na melhora da expansão pulmonar e tóraco-abdominal. Giovanetti et al. ${ }^{17}$ evidenciaram aumento significativo dos volumes mostrando a eficácia do tratamento com ambos os aparelhos. Não foi evidenciada alteração na FR, sendo que estudos anteriores ${ }^{28,29}$ sugerem que a redução da FR promovida pelo espirômetro a volume está associada a um maior tempo inspiratório, proporcionada pela inspiração lenta e profunda, favorável ao desenvolvimento de fluxo aéreo laminar e uniforme. No entanto, esses estudos avaliaram a FR no momento em que os sujeitos utilizavam a espirometria de incentivo, e não após intervenção.

Como limitação do estudo considera-se que um protocolo com maior número de sessões e repetições possa propiciar melhores resultados, sugerindo que o tratamento não deva ser limitado em função do tempo.

\section{CONCLUSÃO}

Tanto o incentivador a fluxo quanto a volume foram eficazes e apresentaram benefícios similares no aumento da força muscular respiratória, função pulmonar e mobilidade tóraco-abdominal, trazendo contribuições no 
âmbito da fisioterapia, especialmente na saúde do idoso. Pelo fácil manuseio e baixo custo, podem ser de grande valia na manutenção da saúde pulmonar do idoso, o que pode reduzir a incidência de disfunções respiratórias, internações hospitalares e ônus ao sistema de saúde.

\section{REFERÊNCIAS}

1. Rebelatto JR, Morelli JGS. Fisioterapia Geriátrica: a prática da assistência do idoso. São Paulo: Manole; 2004.

2. Lima-Costa MF, Barreto SM, Giatti L. Condições de saúde, capacidade funcional, uso de serviços de saúde e gastos com medicamentos da população idosa brasileira: um estudo descritivo baseado na Pesquisa Nacional por Amostra de Domicílios. Cad Saúde Pública. 2003:19(3):735-43

3. Soares LT. Comparação do padrão vocal de idosos com e sem doença pulmonar obstrutiva crônica [dissertação]. São Paulo: Universidade Federal de São Paulo; 2001.

4. Griffith KA, Sherrill DL, Siegel EM, Manolio TA, Bonekat HW, Enright PL. Predictors of loss of lung function in the elderly: the cardiovascular health study. Am J Respir Crit Care Med. 2001;163:61-8.

5. Francisco MSB, Donalisio MR, Barros MBA, César CLG, Carandina L, Goldbaum M. Fatores associados à doença pulmonar em idosos. Rev Saúde Pública. 2006:40(3):428-35.

6. Faria CN [Internet]. Alterações anatômicas e fisiológicas do envelhecimento. Grandes síndromes geriátricas [acesso maio de 2012]. Disponível em: http://www.ciape.org.br/matdidatico/anacristina/ alteracoes_anatomicas.rtf

7. Hristara-Papadopoulou A, Tsanakas J, Diomou G, Papadopoulou O. Current devices of respiratory physiotherapy. Hippokratia. 2008;12(4):211-20.

8. Yamaguti WPS, Sakamoto ET, Panazollo D, Peixoto CC, Cerri GG, Albuquerque ALP. Mobilidade diafragmática durante espirometria de incentivo orientada a fluxo e a volume em indivíduos sadios. J Bras Pneumol. 2010;36(6):738-45.

9. Ide MR, Caromano FA, Dip MAVB, Guerino MR. Exercícios respiratórios na expansibilidade torácica de idosos: exercícios aquáticos e no solo. Fisioter Mov. 2007:20(2):33-40.

10. Rocha CBJ, Araújo S. Avaliação das pressões respiratórias máximas em pacientes renais crônicos nos momentos pré e pós-hemodiálise. J Bras Nefrol. 2010:32(1):107-13.

11. Souza RB. Pressões Respiratórias Estáticas Máximas. J Bras Pneumol. 2002;28(supl 3):155-65.

12. Neder JA, Andreoni S, Lerario MC, Nery LE. Reference values for lung function tests. II. Maximal respiratory pressures and voluntary ventilation. Braz J Med Biol Res.1999; 32(6);719-27.

13. Caldeira VS, Starling CCD, Britto RR, Martins JA, Sampaio RF, Parreira VF. Precisão e acurácia da cirtometria em adultos saudáveis. J Bras Pneumol. 2007:33(5):519-26.
14. Pereira CAC, Neder JA. Diretrizes para Testes de Função Pulmonar. J Bras Pneumol. 2002;28(Suppl 3):1-13.

15. Renault JA, Costa-Val R, Rossetti MB, Houri Neto M. Comparação entre exercícios de respiração profunda e espirometria de incentivo no pós-operatório de cirurgia de revascularização do miocárdio. Rev Bras Cir Cardiovasc. 2009;24(2):165-72.

16. Burneiko RCVM, Melatto T, Padulla SAT, Matta MV, Giacomassi IWS, Sato KT. Efeitos da inspiração fracionada ou incentivador a volume no pós-operatório revascularização do miocárdio. Rev Eletronica Fisioter da FCT/UNESP. 2009;1(1):124-138

17. Giovanetti EA, Boueri CA, Braga KF. Estudo comparativo dos volumes pulmonares e oxigenação após o uso do Respiron e Voldyne no pósoperatório de cirurgia abdominal alta. Reabilitar. 2004;6(25):30-39.

18. Simões RP, Castello V, Auad MA, Dionísio J, Mazzonetto M. Força muscular respiratória e sua relação com a idade em idosos de sessenta a noventa anos. RBCEH. 2010;7(1):52-61.

19. Oyarzún M. Función respiratoria en la senectud. Rev Méd Chile. 2009;137:411-8.

20. Simões RP, Castello V, Auad MA, Dionísio J, Mazzonetto M. Prevalence of reduced respiratory muscle strengh in institucionalized elderly people. São Paulo Med J. 2009:127(2):78-83.

21. Freitas FS, Ibiapina CC, Alvim CG, Britto RR, Parreira VF. Relação entre força de tosse e nível funcional em um grupo de idosos. Rev Bras Fisioter. 2010:4(6):470-6.

22. Fonseca MA, Cader SA, Dantas EHM, Bacelar SC, Silva EB, Leal SMO. Programas de treinamento muscular respiratório: impacto na autonomia funcional de idosos. AMB Rev Assoc Med Bras. 2010:56(6):642-8.

23. Kotz JC. Estudo comparativo do efeito dos incentivadores respiratórios voldyne e respiron sobre a força dos músculos inspiratórios em indivíduos saudáveis. [graduação]. Paraná (PR): Universidade Estadual do Oeste do Paraná; 2005.

24. Weindler J, Kiefer RT. The efficacy of postoperative incentive spirometry is influenced by the device: specific imposed work of breathing. Chest. 2001;119:1858-64.

25. Lunardi AC, Porras DC, Barbosa RC, Paisani DM, Marques da Silva CC, Tanaka C, Carvalho CR. Comparison of distinct incentive spirometers on chest wall volumes, inspiratory muscular activity and thoracoabdominal synchrony in the elderly. Respir Care. 2013 Aug 27. [Epub ahead of print].

26. Guimarães ACA, Pedrini A, Matte DL, Monte FG, Parcias SR. Ansiedade e parâmetros funcionais respiratórios de idosos praticantes de dança. Fisioter Mov. 2011;24(4):683-8.

27. Ho SC, Chiang LL, Cheng HF, Lin HC, Sheng DF, Kuo HP, Lin HC. The Effect of incentive spirometry on chest expansion and breathing work in patients withchronic obstructive airway disease: comparison of two methods. Chang Gung Med J. 2000:23(2):73-9.

28. Parreira VF, Tomich GM, Britto RR, Sampaio RF. Assessment of tidal volume and thoracoabdominal motion using volume and floworiented incentive spirometers in healthy subjects. Braz J Med Biol Res. 2005;38(7):1105-12.

29. Tomich GM, França DC, Diório ACM, Britto RR, Sampaio RF, Parreira VF. Breathing pattern, thoracoabdominal motion and muscular activity during three breathing exercises. Braz J Med Biol Res. 2007;40(10):1409-17. 